Article

\title{
Variational-Like Inequality Problem Involving Generalized Cayley Operator
}

\author{
Zahoor Ahmad Rather ${ }^{1,+}+\mathbb{C}$, Rais Ahmad ${ }^{1,+}$ and Ching-Feng Wen ${ }^{2,3, *,+}+\mathbb{C}$ \\ 1 Department of Mathematics, Aligarh Muslim University, Aligarh 202002, India; \\ zahoorrather348@gmail.com (Z.A.R.); raisain_123@rediffmail.com (R.A.) \\ 2 Center for Fundamental Science, Research Center for Nonlinear Analysis and Optimization, \\ Kaohsiung Medical University, Kaohsiung 80708, Taiwan \\ 3 Department of Medical Research, Kaohsiung Medical University Hospital, Kaohsiung 80708, Taiwan \\ * Correspondence: cfwen@kmu.edu.tw \\ + These authors contributed equally to this work.
}

Citation: Rather, Z.A.; Ahmad, R.; Wen, C.-F. Variational-Like Inequality Problem Involving Generalized Cayley Operator. Axioms 2021, 10, 133.

https://doi.org/10.3390/

axioms10030133

Academic Editor: Wei-Shih Du

Received: 1 June 2021

Accepted: 24 June 2021

Published: 26 June 2021

Publisher's Note: MDPI stays neutral with regard to jurisdictional claims in published maps and institutional affiliations.

Copyright: (c) 2021 by the authors. Licensee MDPI, Basel, Switzerland. This article is an open access article distributed under the terms and conditions of the Creative Commons Attribution (CC BY) license (https:/ / creativecommons.org/licenses/by/ $4.0 /)$.

\begin{abstract}
This article deals with the study of a variational-like inequality problem which involves the generalized Cayley operator. We compare our problem with a fixed point equation, and based on it we construct an iterative algorithm to obtain the solution of our problem. Convergence analysis as well as stability analysis are studied.
\end{abstract}

Keywords: algorithm; solution; stability; sequence; inclusion

\section{Introduction}

The mathematical formalism of a classical variational inequality problem is to find $y \in \mathcal{H}$ such that

$$
\langle T y, x-y\rangle \geq 0, \forall x \in \mathcal{H},
$$

where $\mathcal{H}$ is a Hilbert space and $T: \mathcal{H} \rightarrow \mathcal{H}$ is a nonlinear operator. The concept of variational inequalities was introduced by Stampacchia [1] and Fichera [2], separately. The variational inequality theory has received adequate recognition due to its implementation in a diverse range of problems arising in economics, physics, mathematical finance, structural analysis and in many branches of social, pure and applied sciences, see, for example, in [3-14]. Stampacchia [1] proved that the possible problems related with elliptic equations can be analysed through variational inequalities. Combining auxiliary principle technique and projection operator technique, Lions and Stampacchia [15] studied the existence of solution of variational inequalities.

The variational-like inequalities are the generalized forms of variational inequalities and provide us cogent tools to study many problems of basic and applied sciences. It is obvious that variational inequalities and variational-like inequalities are analogous of fixed point equations. This flipside equivalent formulation plays a significant role in many aspects of variational inequalities and variational-like inequalities. More precisely, this equivalent formulation is used to develop iterative algorithms and to study numerical methods related to variational inequalities and variational-like inequalities, etc.

It is well known that Cayley transform is a mapping between skew-symmetric matrices and special orthogonal matrices. As far as Hilbert spaces are concerned, Cayley transform is a mapping between linear operators. This transform is a homography having applications in real analysis, complex analysis and quaternionic analysis, etc.

As this subject is application oriented, in this paper, we consider a variational-like inequality problem involving generalized Cayley operator. An iterative algorithm is defined to obtain the solution of variational-like inequality problem involving generalized Cayley operator. For more details and recent developments of the subject, we refer to the 
works in [16-29] and the references therein. An existence and convergence result is proved. Stability analysis is also discussed.

\section{Preliminaries}

Let $\mathcal{H}$ be a real Hilbert space with usual norm $\|$.$\| and inner product \langle\cdot, \cdot\rangle$. Let $C B(\mathcal{H})$ be the family of all nonempty closed and bounded subsets of $\mathcal{H}$ and $\mathcal{D}(\cdot, \cdot)$ be the Hausdorff metric on $C B(\mathcal{H})$ defined by

$$
\mathcal{D}(\mathcal{A}, \mathcal{B})=\max \left\{\sup _{x \in \mathcal{A}} d(x, \mathcal{B}), \sup _{y \in \mathcal{B}} d(\mathcal{A}, y)\right\}, \forall \mathcal{A}, \mathcal{B} \in C B(\mathcal{H}),
$$

where $d(x, \mathcal{B})=\inf _{y \in \mathcal{B}} d(x, y)$ and $d(\mathcal{A}, y)=\inf _{x \in \mathcal{A}} d(x, y)$.

The following well known concepts are needed to achieve the goal of this paper.

Definition 1. [30] Let $G: \mathcal{H} \times \mathcal{H} \rightarrow \mathcal{H}$ and $g: \mathcal{H} \rightarrow \mathcal{H}$ be single-valued mappings. Then

(i) $G(.,$.$) is said to be Lipschitz continuous in the first argument, if there exists a constant$ $\lambda_{G_{1}}>0$ such that

$$
\left\|G\left(x_{1}, .\right)-G\left(x_{2}, .\right)\right\| \leq \lambda_{G_{1}}\left\|x_{1}-x_{2}\right\|, \quad \forall x_{1}, x_{2} \in \mathcal{H} .
$$

(ii) $G(.,$.$) is said to be Lipschitz continuous in the second argument, if there exists a constant$ $\lambda_{G_{2}}>0$ such that

$$
\left\|G\left(., y_{1}\right)-G\left(., y_{2}\right)\right\| \leq \lambda_{G_{2}}\left\|y_{1}-y_{2}\right\|, \quad \forall y_{1}, y_{2} \in \mathcal{H} .
$$

(iii) $g: \mathcal{H} \rightarrow \mathcal{H}$ is said to be Lipschitz continuous, if there exists a constant $\lambda_{g}>0$ such that

$$
\|g(x)-g(y)\| \leq \lambda_{g}\|x-y\|, \quad \forall x, y \in \mathcal{H} .
$$

(iv) $g$ is said to be strongly monotone, if there exists a constant $\mu_{g}>0$ such that

$$
\langle g(x)-g(y), x-y\rangle \geq \mu_{g}\|x-y\|^{2}, \forall x, y \in \mathcal{H} .
$$

Definition 2. [30] A multi-valued mapping $F: \mathcal{H} \rightarrow C B(\mathcal{H})$ is said to be $\mathcal{D}$-Lipschitz continuous if there exists a constant $\lambda_{D_{F}}>0$ such that

$$
\mathcal{D}(F(x), F(y)) \leq \lambda_{D_{F}}\|x-y\|, \forall x, y \in \mathcal{H} .
$$

Definition 3. [31,32] A functional $f: \mathcal{H} \times \mathcal{H} \rightarrow R \cup\{+\infty\}$ is said to be 0 -diagonally quasiconcave (in short, $0-D Q C V$ ) in $x$ if for any finite set $\left\{x_{1}, \ldots, x_{n}\right\} \subset \mathcal{H}$ and for any $y=\sum_{i=1}^{n} \lambda_{i} x_{i}$ with $\lambda_{i} \geq 0$ and $\sum_{i=1}^{n} \lambda_{i}=1, \min _{1 \leq i \leq n} f\left(x_{i}, y\right) \leq 0$.

Definition 4. [33] A mapping $\eta: \mathcal{H} \times \mathcal{H} \rightarrow \mathcal{H}$ is said to be

(i) $\delta$-strongly monotone, if there exists a constant $\delta>0$ such that

$$
\langle\eta(x, y), x-y\rangle \geq \delta\|x-y\|^{2}, \forall x, y \in \mathcal{H} .
$$

(ii) $\tau$-Lipschitz continuous, if there exists a constant $\tau>0$ such that

$$
\|\eta(x, y)\| \leq \tau\|x-y\|, \quad \forall x, y \in \mathcal{H} .
$$


Definition 5. [30] Let $\eta: \mathcal{H} \times \mathcal{H} \rightarrow \mathcal{H}$ be a single-valued mapping. A proper convex functional $\phi: \mathcal{H} \rightarrow R \cup\{+\infty\}$ is said to be $\eta$-subdifferentiable at a point $x \in \mathcal{H}$ if there exists a point $f^{*} \in \mathcal{H}$ such that

$$
\phi(y)-\phi(x) \geq\left\langle f^{*}, \eta(y, x)\right\rangle, \forall y \in \mathcal{H},
$$

where $f^{*}$ is called a $\eta$-subgradient of $\phi$ at $x$. The set of all $\eta$-subgradients of $\phi$ at $x$ is denoted by $\partial_{\eta} \phi$. The mapping $\partial_{\eta} \phi: \mathcal{H} \rightarrow 2^{\mathcal{H}}$ defined by

$$
\partial_{\eta} \phi=\left\{f^{*} \in \mathcal{H}: \phi(y)-\phi(x) \geq\left\langle f^{*}, \eta(y, x)\right\rangle, \forall y \in \mathcal{H}\right\}
$$

is called $\eta$-subdifferential of $\phi$.

Lemma 1. [30] Let $X$ be a nonempty convex subset of a topological vector space and $f: X \times X \rightarrow$ $[-\infty,+\infty]$ be such that

(i) for each $x \in X, y \mapsto f(x, y)$ is lower semicontinuous on each compact subset of $X$,

(ii) for each finite set $\left\{x_{1}, \ldots, x_{m}\right\} \subset X$ and for each $y=\sum_{i=1}^{m} \lambda_{i} x_{i}$ with $\lambda_{i} \geq 0$ and $\sum_{i=1}^{m} \lambda_{i}=$ $1, \min _{0 \leq i \leq 1} f\left(x_{i}, y\right) \leq 0$,

(iii) there exists a nonempty compact convex subset $X_{0}$ of $X$ and a nonempty compact subset $K$ of $X$ such that for each $y \in X \backslash K$, there is an $x \in \operatorname{Co}\left(X_{0} \cup\{y\}\right)$ satisfying $f(x, y)>0$.

Then there exists $y^{\prime} \in X$ such that $f\left(x, y^{\prime}\right) \leq 0$, for all $x \in X$.

Theorem 1. [30] Let $\eta: \mathcal{H} \times \mathcal{H} \rightarrow \mathcal{H}$ be continuous and $\delta$-strongly monotone such that $\eta(x, y)=$ $-\eta(y, x)$ for all $x, y \in \mathcal{H}$ and for any given $x \in \mathcal{H}$, the functional $h(y, u)=\langle x-u, \eta(y, u)\rangle$ is $0-D Q C V$ in $y$. Let $\phi: \mathcal{H} \rightarrow R \cup\{+\infty\}$ be a lower semicontinuous, $\eta$-subdifferentiable, proper functional. Then, for any given $\rho>0$ and $x \in \mathcal{H}$, there exist a unique $u \in \mathcal{H}$ such that

$$
\langle u-x, \eta(y, u)\rangle \geq \rho \phi(u)-\rho \phi(y), \forall y \in \mathcal{H} .
$$

That is $u=J_{\rho}^{\partial_{\eta} \phi}(x)$.

Theorem 2. [30] Let $\eta: \mathcal{H} \times \mathcal{H} \rightarrow \mathcal{H}$ be $\delta$-strongly monotone and $\tau$-Lipschitz continuous such that $\eta(x, y)=-\eta(y, x)$ for all $x, y \in \mathcal{H}$ and for any given $x \in \mathcal{H}$, the functional $h(y, u)=$ $\langle x-u, \eta(y, u)\rangle$ is $0-D Q C V$ in $y$. Let $\phi: \mathcal{H} \rightarrow R$ be a lower semicontinuous, $\eta$-subdifferentiable, proper functional, and $\rho>0$ be a arbitrary constant. Then, the $\eta$-proximal mapping $J_{\rho}^{\partial_{\eta} \phi}$ of $\phi$ is $\frac{\tau}{\delta}$-Lipschitz continuous.

Definition 6. [34] Let $S, T: \mathcal{H} \rightarrow \mathcal{H}$ be a single-valued mapping, $x_{0} \in \mathcal{H}$ and

$$
x_{n+1}=S\left(T, x_{n}\right)
$$

defines an iterative sequence which yields a sequence of points $\left\{x_{n}\right\}$ in $\mathcal{H}$. Suppose that $F(T)=$ $\{p \in \mathcal{H}: T p=p\} \neq \varnothing$ and $\left\{x_{n}\right\}$ converges to a fixed point $x^{*}$ of T. Let $\left\{u_{n}\right\} \subset \mathcal{H}$ and

$$
\vartheta_{n}=\left\|u_{n+1}-S\left(T, u_{n}\right)\right\| .
$$

The $\lim _{n \rightarrow \infty} \vartheta_{n}=0$, implies that $u_{n} \rightarrow x^{*}$, and consequently the iterative sequence $\left\{x_{n}\right\}$ is said to be $T$-stable or stable with respect to $T$.

Definition 7. [35] A multi-valued mapping $M: \mathcal{H} \rightarrow C B(\mathcal{H})$ is said to be monotone if, for any $x, y \in \mathcal{H}$

$$
\left\langle u_{1}-u_{2}, x-y\right\rangle \geq 0, \forall u_{1} \in M(x), u_{2} \in M(y)
$$


Definition 8. [35] If $M$ is a maximal monotone mapping, then for a fixed $\rho>0$, the resolvent operator associated with $M$ is defined as

$$
\mathcal{R}_{I, \rho}^{M}(x)=[I+\rho M]^{-1}(x), \forall x \in \mathcal{H} \text {, where } I \text { is the identity operator. }
$$

It is well known that the resolvent operator $\mathcal{R}_{I, \rho}^{M}$ is single-valued.

As the $\eta$-subdifferential operator $\partial_{\eta} \phi$ of $\phi$ is maximal monotone, we define the resolvent operator for a fixed $\rho>0$ as

$$
\mathcal{R}_{I, \rho}^{\partial_{\eta} \phi}(x)=\left[I+\rho \partial_{\eta} \phi\right]^{-1}(x), \forall x \in \mathcal{H}, \text { where I is the identity operator. }
$$

In case, if $\phi: \mathcal{H} \times \mathcal{H} \rightarrow R \cup\{+\infty\}$, then the resolvent operator is defined for a fixed $\rho>0$ as

$$
\mathcal{R}_{I, \rho}^{\partial_{\eta} \phi(. . .)}(x)=\left[I+\rho \partial_{\eta} \phi(., .)\right]^{-1}(x), \forall x \in \mathcal{H} \text {, where I is the identity operator. }
$$

Definition 9. [36] The generalized Cayley operator $C_{I, \rho}^{\partial_{\eta} \phi(. .)}$ is defined as

$$
C_{I, \rho}^{\partial_{\eta} \phi(. . .)}(x)=\left[2 R_{I, \rho}^{\partial_{\eta} \phi(. .)}-I\right](x), \text { for all } x \in \mathcal{H} \text { and for a fixed } \rho>0,
$$

where $R_{I, \rho}^{\partial_{\eta} \phi(. . .)}$ is defined by (2).

Lemma 2. The generalized Cayley operator $C_{I, \rho}^{\partial_{\eta} \phi(., x)}$ is $\frac{2 \tau+\delta}{\delta}$-Lipschitz continuous.

Proof. As the resolvent operator $R_{I, \rho}^{\partial_{\eta} \phi(., x)}$ is $\frac{\tau}{\delta}$-Lipschitz continuous, we have

$$
\begin{aligned}
\left\|C_{I, \rho}^{\partial_{\eta} \phi\left(., x_{n}\right)}\left(x_{n}\right)-C_{I, \rho}^{\partial_{\eta} \phi\left(., x_{n}\right)}\left(x_{n-1}\right)\right\|= & \|\left[2 R_{I, \rho}^{\partial_{\eta} \phi\left(., x_{n}\right)}\left(x_{n}\right)-I\left(x_{n}\right)\right]- \\
& {\left[2 R_{I, \rho}^{\partial_{\eta} \phi\left(., x_{n}\right)}\left(x_{n-1}\right)-I\left(x_{n-1}\right)\right] \| } \\
\leq & \left\|2 R_{I, \rho}^{\partial_{\eta} \phi\left(., x_{n}\right)}\left(x_{n}\right)-2 R_{I, \rho}^{\partial_{\eta} \phi\left(., x_{n}\right)}\left(x_{n-1}\right)\right\| \\
& +\left\|x_{n}-x_{n-1}\right\| \\
\leq & 2 \frac{\tau}{\delta}\left\|x_{n}-x_{n-1}\right\|+\left\|x_{n}-x_{n-1}\right\| \\
= & \left(\frac{2 \tau+\delta}{\delta}\right)\left\|x_{n}-x_{n-1}\right\| .
\end{aligned}
$$

Lemma 3. [34] Let $\left\{a_{n}\right\}_{n=0}^{\infty},\left\{b_{n}\right\}_{n=0}^{\infty}$ be sequences of nonnegative numbers and $0 \leq q<1$, so that

$$
a_{n+1} \leq q a_{n}+b_{n}, \text { for all } n \geq 0
$$

If $\lim _{n \rightarrow \infty} b_{n}=0$, then $\lim _{n \rightarrow \infty} a_{n}=0$.

\section{Formulation of Problem and Fixed Point Formulation}

Let $\phi: \mathcal{H} \times \mathcal{H} \rightarrow R \cup\{+\infty\}$ be a convex, proper, lower semicontinuous functional and $\eta: \mathcal{H} \times \mathcal{H} \rightarrow H$ be a single-valued mapping. Let $F: \mathcal{H} \rightarrow C B(\mathcal{H})$ be a multivalued mapping and $G: \mathcal{H} \times \mathcal{H} \rightarrow \mathcal{H}, g: \mathcal{H} \rightarrow \mathcal{H}$ be the single-valued mappings such that $g(\mathcal{H}) \cap \operatorname{dom} \partial_{\eta} \phi(., x) \neq \phi$. Suppose $C_{I, \rho}^{\partial_{\eta} \phi(., x)}: \mathcal{H} \rightarrow \mathcal{H}$ is the generalized Cayley operator. We study the following variational-like inequality problem involving generalized Cayley operator. 
Find $x \in \mathcal{H}, t \in F(x)$ such that $g(x) \in \partial_{\eta} \phi(., x)$ and

$$
\left\langle G(t, x)-C_{I, \rho}^{\partial_{\eta} \phi(., x)}(x), \eta(y, g(x))\right\rangle \geq \phi(g(x), x)-\phi(y, x), \quad \forall y \in \mathcal{H} .
$$

We list below some special cases of variational-like inequality problem involving generalized Cayley operator (4).

Special cases :

Case 1: If $G(t, x)=T(x), C_{I, \rho}^{\partial_{\eta} \phi(., x)}(x)=A(x)$, where $T, A: \mathcal{H} \rightarrow \mathcal{H}$, then problem (4) reduces to a general quasi-variational-like inclusion problem:

Find $x \in \mathcal{H}$ such that $g(x) \in \operatorname{dom} \partial_{\eta} \phi(., x)$

$$
\langle T(x)-A(x), \eta(y, g(x))\rangle \geq \phi(g(x), x)-\phi(y, x), \forall y \in \mathcal{H} .
$$

Problem (5) was introduced and studied by Ding and Luo [37].

Case 2: If $G(t, x)=T(x), C_{I, \rho}^{\partial_{\eta} \phi(., x)}(x)=A(x), \phi(x, y)=\phi(x), \partial_{\eta} \phi(., x)=\partial \phi(x)$ and $\eta(y, x)=y-x$ for all $x, y \in \mathcal{H}$, then problem (4) reduces to the following variational inclusion problem:

Find $x \in \mathcal{H}$ such that $g(x) \in \operatorname{dom} \partial \phi(x)$

$$
\langle T(x)-A(x), y-g(x)\rangle \geq \phi(g(x))-\phi(y), \quad \forall y \in \mathcal{H} .
$$

Problem (6) was introduced and studied by Hassouni and Moudafi [38] and Huang [39].

Case 3: If $G(t, x)=T(x), C_{I, \rho}^{\partial_{\eta} \phi(., x)}(x)=A(x), \phi(x, y)=\phi(x)$, also if $K: \mathcal{H} \rightarrow 2^{\mathcal{H}}$ be a given multi-valued mapping such that each $K(x)$ is a closed convex subset of $\mathcal{H}$ (or $K(x)=m(x)+K$ where $m: \mathcal{H} \rightarrow \mathcal{H}$ and $K$ is a closed convex subset of $\mathcal{H})$ and if $\eta(x, y)=x-y$ for all $x, y \in \mathcal{H}, \phi: \mathcal{H} \times \mathcal{H} \rightarrow \mathcal{H}$ is defined by $\phi(x, y)=I_{K(y)}(x), \forall x, y \in \mathcal{H}$,

where $I_{K(y)}(x)$ is the indicator function of $K(y)$, that is,

$$
I_{K(y)}(x)= \begin{cases}0 & \text { if } x \in K(y) \\ +\infty & \text { otherwise }\end{cases}
$$

then problem (4) reduces to the following strongly nonlinear quasi-variational inequality problem:

Find $x \in \mathcal{H}$ such that $g(x) \in K(x)$ and

$$
\langle T(x)-A(x), y-g(x)\rangle \geq 0, \forall y \in K(x) .
$$

Problem (7) includes various classes of variational inequalities, quasi-variational inequalities, complementarity and quasi-complementarity problems, studied previously by many authors, see [40,41].

It is shown below that problem (4) is equivalent to a fixed point equation.

Lemma 4. The variational-like inequality problem involving generalized Cayley operator (4) has a solution $x \in \mathcal{H}, t \in F(x)$ if and only if the following equation is satisfied:

$$
g(x)=\mathcal{R}_{I, \rho}^{\partial_{\eta} \phi(., x)}\left[g(x)-\rho\left(G(t, x)-C_{I, \rho}^{\partial_{\eta} \phi(., x)}(x)\right)\right],
$$

where $\mathcal{R}_{I, \rho}^{\partial_{\eta} \phi(., x)}=\left[I+\rho \partial_{\eta} \phi(., x)\right]^{-1}$ is the resolvent operator.

Proof. Let $x \in \mathcal{H}, t \in F(x)$ satisfy the Equation (8), that is

$$
g(x)=\mathcal{R}_{I, \rho}^{\partial_{\eta} \phi(., x)}\left\{g(x)-\rho\left(G(t, x)-C_{I, \rho}^{\partial_{\eta} \phi(., x)}(x)\right)\right\} .
$$


Using the definition of resolvent operator, the above inequality holds if and only if

$$
\begin{aligned}
g(x)-\rho\left(G(t, x)-C_{I, \rho}^{\partial_{\eta}(., x)}(x)\right) & \in g(x)+\rho \partial_{\eta} \phi(g(x), x), \\
\text { that is, } \quad C_{I, \rho}^{\partial_{\eta}(., x)}(x)-G(t, x) & \in \partial_{\eta} \phi(g(x), x) .
\end{aligned}
$$

Applying $\eta$-subdifferentiability of $\partial_{\eta}(., x)$, the above relation holds if and only if

$$
\left\langle C_{I, \rho}^{\partial_{\eta} \phi(., x)}(x)-G(t, x), \eta(y, g(x))\right\rangle \leq \phi(y, x)-\phi(g(x), x) .
$$

Thus, we have

$$
\left\langle G(t, x)-C_{I, \rho}^{\partial_{\eta} \phi(., x)}(x), \eta(y, g(x))\right\rangle \geq \phi(g(x), x)-\phi(y, x) .
$$

The result follows.

\section{Iterative Algorithm and Convergence Result}

Using Lemma 4, we construct an iterative algorithm to obtain the solution of problem (4).

\section{Algorithm 1}

For initial elements $x_{0} \in \mathcal{H}, t_{0} \in F\left(x_{0}\right)$ such that $g\left(x_{0}\right) \in \partial_{\eta} \phi\left(., x_{0}\right)$, let

$$
x_{1}=(1-\lambda) x_{0}+\lambda\left[x_{0}-g\left(x_{0}\right)+\mathcal{R}_{I, \rho}^{\partial_{\eta} \phi\left(., x_{0}\right)}\left[g\left(x_{0}\right)-\rho\left(G\left(t_{0}, x_{0}\right)-C_{I, \rho}^{\partial_{\eta} \phi\left(., x_{0}\right)}\left(x_{0}\right)\right]\right] .\right.
$$

As $t_{0} \in F\left(x_{0}\right) \in C B(\mathcal{H})$, by Nadler [42], there exists $t_{1} \in F\left(x_{1}\right)$ such that

$$
\left\|t_{0}-t_{1}\right\| \leq \mathcal{D}\left(F\left(x_{0}\right), F\left(x_{1}\right)\right) .
$$

Let

$$
x_{2}=(1-\lambda) x_{1}+\lambda\left[x_{1}-g\left(x_{1}\right)+\mathcal{R}_{I, \rho}^{\partial_{\eta} \phi\left(., x_{1}\right)}\left[g\left(x_{1}\right)-\rho\left(G\left(t_{1}, x_{1}\right)-C_{I, \rho}^{\partial_{\eta} \phi\left(., x_{1}\right)}\left(x_{1}\right)\right)\right]\right] .
$$

As $t_{1} \in F\left(x_{1}\right) \in C B(\mathcal{H})$, there exists $t_{2} \in F\left(x_{2}\right)$ such that

$$
\left\|t_{1}-t_{2}\right\| \leq \mathcal{D}\left(F\left(x_{1}\right), F\left(x_{2}\right)\right) .
$$

Based on above observations, we compute the sequences $\left\{x_{n}\right\}$ and $\left\{t_{n}\right\}$ as

$$
x_{n+1}=(1-\lambda) x_{n}+\lambda\left[x_{n}-g\left(x_{n}\right)+\mathcal{R}_{I, \rho}^{\partial_{\eta} \phi\left(., x_{n}\right)}\left[g\left(x_{n}\right)-\rho\left(G\left(t_{n}, x_{n}\right)-C_{I, \rho}^{\partial_{\eta} \phi\left(., x_{n}\right)}\left(x_{n}\right)\right]\right],\right.
$$

and for $t_{n} \in F\left(x_{n}\right), t_{n+1} \in F\left(x_{n+1}\right)$,

$$
\left\|t_{n}-t_{n+1}\right\| \leq \mathcal{D}\left(F\left(x_{n}\right), F\left(x_{n+1}\right)\right),
$$

for $n=0,1,2, \ldots$ and $0<\lambda \leq 1$.

Theorem 3. Let $G, \eta: \mathcal{H} \times \mathcal{H} \rightarrow \mathcal{H} ; g: \mathcal{H} \rightarrow \mathcal{H}$ be single-valued mappings such that $g$ : $\mathcal{H} \rightarrow \mathcal{H}$ be Lipschitz continuous and strongly monotone mapping with constants $\lambda_{g}$ and $\delta_{g}$, respectively; $G$ is Lipschitz continuous in the first and second arguments with constants $\lambda_{G_{1}}$ and $\lambda_{G_{2}}$, respectively; $\eta$ be $\delta$-strongly monotone and $\tau$-Lipschitz continuous such that $\eta(x, y)=$ $-\eta(y, x)$, for all $x, y \in \mathcal{H}$. Let the functional $h(y, u)=\langle x-u, \eta(y, u)\rangle$ is $0-D Q C V$ in $y$ and $\phi: \mathcal{H} \times \mathcal{H} \rightarrow R$ be such that for each fixed $y \in \mathcal{H}, \phi(., y)$ is lower-semicontinuous, $\eta$ subdifferentiable proper functional satisfying $g(\mathcal{H}) \cap$ dom $_{\eta} \phi(., y) \neq \phi$, where $\partial_{\eta} \phi(.,$.$) denotes$ the $\eta$-subdifferential of $\phi(.,$.$) . Suppose that C_{I, \rho}^{\partial_{n} \phi(., x)}: \mathcal{H} \rightarrow \mathcal{H}$ is the Cayley operator such that 
$C_{I, \rho}^{\partial_{n} \phi(\cdot, x)}$ is Lipschitz continuous with constant $\left(\frac{2 \tau+\delta}{\delta}\right)$. Let $F: \mathcal{H} \rightarrow C B(\mathcal{H})$ be multi-valued mapping such that $F$ is $\mathcal{D}$-Lipschitz continuous with constant $\lambda_{\mathcal{D}_{F}}$. Suppose that the following conditions are satisfied:

$$
\begin{aligned}
& \left\|\mathcal{R}_{I, \rho}^{\partial_{\eta} \phi(., x)}(z)-\mathcal{R}_{I, \rho}^{\partial_{\eta} \phi(., y)}(z)\right\| \leq \mu^{\prime}\|x-y\| \\
\text { and } \quad & \left\|C_{I, \rho}^{\partial_{\eta} \phi(., x)}(z)-C_{I, \rho}^{\partial_{n} \phi(., y)}(z)\right\| \leq \mu^{\prime \prime}\|x-y\| .
\end{aligned}
$$

Furthermore, if the following condition holds:

$$
\left|\lambda_{g}-\frac{\delta^{2}\left(\rho \frac{\tau^{2}}{\delta^{2}}\left(\theta_{1}+\theta_{2}\right)+\frac{\tau \mu^{\prime}}{\delta}-\frac{\tau}{\delta}\right)}{\delta^{2}-\tau^{2}}\right|<\delta^{2} \frac{\sqrt{\left(\rho \frac{\tau^{2}}{\delta^{2}}\left(\theta_{1}+\theta_{2}\right)+\frac{\tau \mu^{\prime}}{\delta}-\frac{\tau}{\delta}\right)^{2}-\frac{\delta^{2}-\tau^{2}}{\delta^{2}}(\Theta)}}{\delta^{2}-\tau^{2}},
$$

where $\Theta=2 \frac{\rho \tau}{\delta}\left(\theta_{1}+\theta_{2}\right)-\frac{\tau^{2} \rho^{2}}{\delta^{2}}\left(\theta_{1}+\theta_{2}\right)^{2}-2 \frac{\tau \mu^{\prime} \rho}{\delta}\left(\theta_{1}+\theta_{2}\right)-2 \delta_{g}-\mu^{\prime 2}+2 \mu^{\prime}$, $\theta_{1}=\left(\lambda_{G_{1}} \lambda_{D_{F}}+\lambda_{G_{2}}\right), \theta_{2}=\left(\frac{2 \tau+\delta}{\delta}+\mu^{\prime \prime}\right)$ and $\lambda_{g}^{2}>\left(2 \delta_{g}-1\right)$.

Then, the sequences $\left\{x_{n}\right\}$ and $\left\{t_{n}\right\}$ generated by Algorithm 1 converge strongly to the solution $x$ and $t$ of variational-like inequality problem involving generalized Cayley operator (4), respectively.

Proof. Using (9) of Algorithm 1, we have

$$
\begin{aligned}
\| x_{n+1}- & x_{n} \| \\
= & \|(1-\lambda) x_{n}+\lambda\left[x_{n}-g\left(x_{n}\right)+\mathcal{R}_{I, \rho}^{\partial_{\eta} \phi\left(., x_{n}\right)}\left[g\left(x_{n}\right)-\rho\left[G\left(t_{n}, x_{n}\right)-\left(C_{I, \rho}^{\partial_{\eta} \phi\left(., x_{n}\right)}\right)\right]\right]\right. \\
& -\left[(1-\lambda) x_{n-1}+\lambda\left\{x_{n-1}-g\left(x_{n-1}\right)+\mathcal{R}_{I, \rho}^{\partial_{\eta} \phi\left(., x_{n-1}\right)}\left[g\left(x_{n-1}\right)-\rho\left(G\left(t_{n-1}, x_{n-1}\right)\right.\right.\right.\right. \\
& \left.\left.\left.-C_{I, \rho}^{\partial_{\eta} \phi\left(., x_{n-1}\right)}\left(x_{n-1}\right)\right)\right\}\right] \| \\
= & \|(1-\lambda)\left(x_{n}-x_{n-1}\right)+\lambda\left[x_{n}-x_{n-1}-\left(g\left(x_{n}\right)-g\left(x_{n-1}\right)\right)\right] \\
& +\lambda\left[\mathcal { R } _ { I , \rho } ^ { \partial _ { \eta } \phi ( . , x _ { n } ) } \left\{g\left(x_{n}\right)-\rho\left(G\left(t_{n}, x_{n}\right)-C_{I, \rho}^{\partial_{\eta} \phi\left(., x_{n}\right)}\left(x_{n}\right)\right\}-\mathcal{R}_{I, \rho}^{\partial_{\eta} \phi\left(., x_{n-1}\right)}\left\{g\left(x_{n-1}\right)\right.\right.\right. \\
& -\rho\left(G\left(t_{n-1}, x_{n-1}\right)-C_{I, \rho}^{\partial_{\eta} \phi\left(., x_{n-1}\right)}\left(x_{n-1}\right)\right] \| \\
\leq & (1-\lambda)\left\|x_{n}-x_{n-1}\right\|+\lambda \| x_{n}-x_{n-1}-\left(g\left(x_{n}\right)-g\left(x_{n-1}\right) \|\right. \\
& +\lambda \| \mathcal{R}_{I, \rho}^{\partial_{\eta} \phi\left(., x_{n}\right)}\left\{g\left(x_{n}\right)-\rho\left(G\left(t_{n}, x_{n}\right)-C_{I, \rho}^{\partial_{\eta} \phi\left(., x_{n}\right)}\left(x_{n}\right)\right)\right\}-\mathcal{R}_{I, \rho}^{\partial_{\eta} \phi\left(., x_{n-1}\right)}\left\{g\left(x_{n-1}\right)\right. \\
& -\rho\left(G\left(t_{n-1}, x_{n-1}\right)-C_{I, \rho}^{\partial_{\eta} \phi\left(., x_{n-1}\right)}\left(x_{n-1}\right)\right\} \| .
\end{aligned}
$$

As $g$ is Lipschitz continuous with constant $\lambda_{g}$ and strongly monotone with constant $\delta_{g}$, by using technique of [35], we have

$$
\| x_{n}-x_{n-1}-\left(g\left(x_{n}\right)-g\left(x_{n-1}\right)\left\|\leq \sqrt{1-2 \delta_{g}+\lambda_{g}^{2}}\right\| x_{n}-x_{n-1} \|,\right.
$$

where $\lambda_{g}^{2}>\left(2 \delta_{g}-1\right)$.

As the resolvent operator $R_{I, \rho}^{\partial_{\eta} \phi(, x)}$ is Lipschitz continuous with constant $\frac{\tau}{\delta}$ and using condition (11), we have $\| \mathcal{R}_{I, \rho}^{\partial_{\eta} \phi\left(., x_{n}\right)}\left\{g\left(x_{n}\right)-\rho\left(G\left(t_{n}, x_{n}\right)-C_{I, \rho}^{\partial_{\eta} \phi\left(., x_{n}\right)}\left(x_{n}\right)\right)\right\}-\mathcal{R}_{I, \rho}^{\partial_{\eta} \phi\left(., x_{n-1}\right)}\left\{g\left(x_{n-1}\right)-\right.$
$\rho\left(G\left(t_{n-1}, x_{n-1}\right)-C_{I, \rho}^{\partial_{\eta} \phi\left(., x_{n-1}\right)}\left(x_{n-1}\right)\right\} \|$ 


$$
\begin{aligned}
\leq & \| \mathcal{R}_{I, \rho}^{\partial_{\eta} \phi\left(., x_{n}\right)}\left\{g\left(x_{n}\right)-\rho\left(G\left(t_{n}, x_{n}\right)-C_{I, \rho}^{\partial_{\eta} \phi\left(., x_{n}\right)}\left(x_{n}\right)\right)\right\} \\
- & \mathcal{R}_{I, \rho}^{\partial_{\eta} \phi\left(., x_{n}\right)}\left\{g\left(x_{n-1}\right)-\rho\left(G\left(t_{n-1}, x_{n-1}\right)-C_{I, \rho}^{\partial_{\eta} \phi\left(., x_{n-1}\right)}\left(x_{n-1}\right)\right)\right\} \\
+ & \mathcal{R}_{I, \rho}^{\partial_{\eta} \phi\left(., x_{n}\right)}\left\{g\left(x_{n-1}\right)-\rho\left(G\left(t_{n-1}, x_{n-1}\right)-C_{I, \rho}^{\partial_{\eta} \phi\left(., x_{n-1}\right)}\left(x_{n-1}\right)\right\}\right. \\
- & \mathcal{R}_{I, \rho}^{\partial_{\eta} \phi\left(., x_{n-1}\right)}\left\{g\left(x_{n-1}\right)-\rho\left(G\left(t_{n-1}, x_{n-1}\right)-C_{I, \lambda}^{\partial_{\eta} \phi\left(., x_{n-1}\right)}\left(x_{n-1}\right)\right\} \|\right. \\
\leq & \left.\frac{\tau}{\delta} \| g\left(x_{n}\right)-\rho\left(G\left(t_{n}, x_{n}\right)\right)-C_{I, \rho}^{\partial_{\eta} \phi\left(., x_{n}\right)}\left(x_{n}\right)\right\}-\left\{g\left(x_{n-1}\right)\right. \\
& -\rho\left(G\left(t_{n-1}, x_{n-1}\right)-C_{I, \rho}^{\partial_{\eta} \phi\left(., x_{n-1}\right)}\left(x_{n-1}\right)\right)\left\|+\mu^{\prime}\right\| x_{n}-x_{n-1} \| .
\end{aligned}
$$

We evaluate,

$$
\begin{aligned}
& \left\|\left(g\left(x_{n}\right)-g\left(x_{n-1}\right)\right)-\rho\left\{\left(G\left(t_{n}, x_{n}\right)-G\left(t_{n-1}, x_{n-1}\right)\right)-\left(C_{I, \rho}^{\partial \phi\left(., x_{n}\right)}\left(x_{n}\right)-C_{I, \rho}^{\partial \phi\left(., x_{n-1}\right)}\left(x_{n-1}\right)\right)\right\}\right\| \\
& \leq\left\|g\left(x_{n}\right)-g\left(x_{n-1}\right)\right\|+\rho\left\|\left(G\left(t_{n}, x_{n}\right)-G\left(t_{n-1}, x_{n-1}\right)\right)\right\| \\
& \left.+\rho \|\left(C_{I, \rho}^{\partial_{\eta} \phi\left(., x_{n}\right)}\left(x_{n}\right)-C_{I, \rho}^{\partial_{\eta} \phi\left(., x_{n-1}\right)}\left(x_{n-1}\right)\right)\right\} \| .
\end{aligned}
$$

Using Lipschitz continuity of $G$ in both the arguments, we get $\left\|G\left(t_{n}, x_{n}\right)-G\left(t_{n-1}, x_{n-1}\right)\right\|$

$$
\begin{aligned}
& =\left\|G\left(t_{n}, x_{n}\right)-G\left(t_{n-1}, x_{n}\right)+G\left(t_{n-1}, x_{n}\right)-G\left(t_{n-1}, x_{n-1}\right)\right\| \\
& =\left\|G\left(t_{n}, x_{n}\right)-G\left(t_{n-1}, x_{n}\right)\right\|+\left\|G\left(t_{n-1}, x_{n}\right)-G\left(t_{n-1}, x_{n-1}\right)\right\| \\
& \leq \lambda_{G_{1}}\left\|t_{n}-t_{n-1}\right\|+\lambda_{G_{2}}\left\|x_{n}-x_{n-1}\right\| .
\end{aligned}
$$

Applying (10) of Algorithm 1 and $\mathcal{D}$-Lipshitz continuity of $F$, we have

$$
\left\|t_{n}-t_{n-1}\right\| \leq \mathcal{D}\left(F\left(x_{n}\right), F\left(x_{n-1}\right) \leq \lambda_{D_{F}}\left\|x_{n}-x_{n-1}\right\| .\right.
$$

Combinings (18) and (19), we have

$$
\begin{aligned}
\left\|G\left(t_{n}, x_{n}\right)-G\left(t_{n-1}, x_{n-1}\right)\right\| & \leq \lambda_{G_{1}} \lambda_{D_{F}}\left\|x_{n}-x_{n-1}\right\|+\lambda_{G_{2}}\left\|x_{n}-x_{n-1}\right\| \\
& =\left(\lambda_{G_{1}} \lambda_{D_{F}}+\lambda_{G_{2}}\right)\left\|x_{n}-x_{n-1}\right\| \\
& =\theta_{1}\left\|x_{n}-x_{n-1}\right\|
\end{aligned}
$$

where $\theta_{1}=\left(\lambda_{G_{1}} \lambda_{D_{F}}+\lambda_{G_{2}}\right)$.

As $C_{I, \rho}^{\partial_{\eta} \phi(., x)}$ is Lipschitz continuous with constant $\frac{2 \tau+\delta}{\delta}$ and using condition (12), we have

$$
\begin{aligned}
& \|\left(C_{I, \rho}^{\partial_{\eta} \phi\left(., x_{n}\right)}\left(x_{n}\right)-\left(C_{I, \rho}^{\partial_{\eta} \phi\left(., x_{n-1}\right)}\left(x_{n-1}\right)\right) \|\right. \\
& =\left\|\left(C_{I, \rho}^{\partial_{\eta} \phi\left(., x_{n}\right)}\left(x_{n}\right)-C_{I, \rho}^{\partial_{\eta} \phi\left(., x_{n}\right)}\left(x_{n-1}\right)+C_{I, \rho}^{\partial_{\eta} \phi\left(., x_{n}\right)}\left(x_{n-1}\right)-C_{I, \rho}^{\partial_{\eta} \phi\left(., x_{n-1}\right)}\left(x_{n-1}\right)\right)\right\| \\
& \leq\left\|\left(C_{I, \rho}^{\partial_{\eta} \phi\left(., x_{n}\right)}\left(x_{n}\right)-C_{I, \rho}^{\partial_{\eta} \phi\left(., x_{n}\right)}\left(x_{n-1}\right)\|+\| C_{I, \rho}^{\partial_{\eta} \phi\left(., x_{n}\right)}\left(x_{n-1}\right)-C_{I, \rho}^{\partial_{\eta} \phi\left(., x_{n-1}\right)}\left(x_{n-1}\right)\right)\right\| \\
& \leq\left(\frac{2 \tau+\delta}{\delta}\right)\left\|x_{n}-x_{n-1}\right\|+\mu^{\prime \prime}\left\|x_{n}-x_{n-1}\right\| \\
& =\left(\frac{2 \tau+\delta}{\delta}+\mu^{\prime \prime}\right)\left\|x_{n}-x_{n-1}\right\| \\
& =\theta_{2}\left\|x_{n}-x_{n-1}\right\|,
\end{aligned}
$$

where $\theta_{2}=\left(\frac{2 \tau+\delta}{\delta}+\mu^{\prime \prime}\right)$. 
Using Lipschitz continuity of $g$, (20) and (21), (17) becomes

$$
\begin{aligned}
\|\left(g\left(x_{n}\right)-g\left(x_{n-1}\right)\right)-\rho\left\{\left(G\left(t_{n}, x_{n}\right)\right.\right. & \left.\left.-G\left(t_{n-1}, x_{n-1}\right)\right)-\left(C_{I, \rho}^{\partial_{\eta} \phi\left(., x_{n}\right)}\left(x_{n}\right)-C_{I, \rho}^{\partial_{\eta} \phi\left(., x_{n-1}\right)}\left(x_{n-1}\right)\right)\right\} \| \\
& \leq \lambda_{g}\left\|x_{n}-x_{n-1}\right\|+\rho \theta_{1}\left\|x_{n}-x_{n-1}\right\|+\rho \theta_{2}\left\|x_{n}-x_{n-1}\right\| \\
& =\left(\lambda_{g}+\rho \theta_{1}+\rho \theta_{2}\right)\left\|x_{n}-x_{n-1}\right\| .
\end{aligned}
$$

Using (22), (16) becomes

$$
\begin{gathered}
\| \mathcal{R}_{I, \rho}^{\partial_{\eta} \phi\left(., x_{n}\right)}\left\{g\left(x_{n}\right)-\rho\left(G\left(t_{n}, x_{n}\right)-C_{I, \rho}^{\partial_{\eta} \phi\left(., x_{n}\right)}\left(x_{n}\right)\right)\right\}-\mathcal{R}_{I, \rho}^{\partial_{\eta} \phi\left(., x_{n-1}\right)}\left\{g\left(x_{n-1}\right)-\right. \\
\rho\left(G\left(t_{n-1}, x_{n-1}\right)-C_{I, \rho}^{\partial_{\eta} \phi\left(., x_{n-1}\right)}\left(x_{n-1}\right)\right\} \| \\
\leq\left[\frac{\tau}{\delta}\left(\lambda_{g}+\rho \theta_{1}+\rho \theta_{2}\right)+\mu^{\prime}\right]\left\|x_{n}-x_{n-1}\right\| .
\end{gathered}
$$

Using (15) and (23), (14) becomes

$$
\begin{aligned}
\left\|x_{n+1}-x_{n}\right\| \leq & (1-\lambda)\left\|x_{n}-x_{n-1}\right\|+\lambda \sqrt{1-2 \delta_{g}+\lambda_{g}^{2}}\left\|x_{n}-x_{n-1}\right\| \\
& +\lambda\left(\frac{\tau}{\delta} \lambda\left(\lambda_{g}+\rho \theta_{1}+\rho \theta_{2}\right)+\mu^{\prime}\right)\left\|x_{n}-x_{n-1}\right\| \\
= & \zeta(\theta)\left\|x_{n}-x_{n-1}\right\| .
\end{aligned}
$$

where $\zeta(\theta)=\left[(1-\lambda)+\lambda \sqrt{1-2 \delta_{g}+\lambda_{g}^{2}}+\lambda\left(\frac{\tau}{\delta}\left(\lambda_{g}+\rho \theta_{1}+\rho \theta_{2}\right)+\mu^{\prime}\right)\right]$.

It follows from condition (13) that $\zeta(\theta)<1$ and consequently $\left\{x_{n}\right\}$ is a Cauchy sequence and hence there exist $x \in \mathcal{H}$ such that $x_{n} \rightarrow x$. From (19), it follows that $\left\{t_{n}\right\}$ is also a Cauchy sequence and hence $t_{n} \rightarrow t \in F(x)$. This completes the proof.

\section{Stability Analysis}

This component deals with the stability analysis of Iterative Algorithm 1.

Theorem 4. If all the mappings and conditions in Theorem 3 remain the same, then iterative scheme 4.1 is $\mathcal{R}_{I, \rho}^{\partial_{\eta} \phi}$-stable.

Proof. Let us consider a sequence $\left\{u_{n}\right\}$ in $\mathcal{H}$ such that

$$
u_{n+1}=(1-\lambda) u_{n}+\lambda\left[u_{n}-g\left(u_{n}\right)+\mathcal{R}_{I, \rho}^{\partial_{\eta} \phi\left(., u_{n}\right)}\left\{g\left(u_{n}\right)-\rho\left(G\left(s_{n}, u_{n}\right)-C_{I, \rho}^{\partial_{\eta} \phi\left(., u_{n}\right)}\left(u_{n}\right)\right\}\right], s_{n} \in F\left(u_{n}\right) .\right.
$$

Also let

$$
\chi_{n}=\| u_{n+1}-\left\{(1-\lambda) u_{n}+\lambda\left\{u_{n}-g\left(u_{n}\right)+\mathcal{R}_{I, \rho}^{\partial_{\eta} \phi\left(., u_{n}\right)}\left\{g\left(u_{n}\right)-\rho\left(G\left(s_{n}, u_{n}\right)-C_{I, \rho}^{\partial_{\eta} \phi\left(., u_{n}\right)}\left(u_{n}\right)\right)\right\}\right\} \| .\right.
$$

and

$$
x^{*}=(1-\lambda) x^{*}+\lambda\left[x^{*}-g\left(x^{*}\right)+\mathcal{R}_{I, \rho}^{\partial_{\eta} \phi\left(., x^{*}\right)}\left\{g\left(x^{*}\right)-\rho\left(G\left(t^{*}, x^{*}\right)-C_{I, \rho}^{\partial_{\eta} \phi\left(., x^{*}\right)}\left(x^{*}\right)\right)\right\}\right] .
$$

Using (24) and (25), we obtain 


$$
\begin{aligned}
\| u_{n+1}- & x^{*} \| \\
= & \| u_{n+1}-\left\{(1-\lambda) u_{n}+\lambda\left\{u_{n}-g\left(u_{n}\right)+\mathcal{R}_{I, \rho}^{\partial_{\eta} \phi\left(., u_{n}\right)}\left\{g\left(u_{n}\right)-\rho\left(G\left(s_{n}, u_{n}\right)\right)\right.\right.\right. \\
- & \left.\left.\left.C_{I, \rho}^{\partial_{\eta} \phi\left(., u_{n}\right)}\left(u_{n}\right)\right)\right\}\right\}+\left\{(1-\lambda) u_{n}+\lambda\left\{u_{n}-g\left(u_{n}\right)+\mathcal{R}_{I, \rho}^{\partial_{\eta} \phi\left(., u_{n}\right)}\left\{g\left(u_{n}\right)-\rho\left(G\left(s_{n}, u_{n}\right)\right.\right.\right.\right. \\
- & \left.\left.\left.C_{I, \rho}^{\partial_{\eta} \phi\left(., u_{n}\right)}\left(u_{n}\right)\right)\right\}\right\}-(1-\lambda) x^{*} \\
+ & \lambda\left[x^{*}-g\left(x^{*}\right)+\mathcal{R}_{I, \rho}^{\partial_{\eta} \phi\left(., x^{*}\right)}\left\{x^{*}-g\left(x^{*}\right)-\rho\left(G\left(t^{*}, x^{*}\right)-C_{I, \rho}^{\partial_{\eta} \phi\left(., x^{*}\right)}\left(x^{*}\right)\right)\right\}\right] \| \\
= & \| u_{n+1}-\left\{(1-\lambda) u_{n}+\lambda\left\{u_{n}-g\left(u_{n}\right)+\mathcal{R}_{I, \rho}^{\partial_{\eta} \phi\left(., u_{n}\right)}\left\{g\left(u_{n}\right)-\rho\left(G\left(s_{n}, u_{n}\right)\right)\right.\right.\right. \\
& \left.\left.\left.-C_{I, \rho}^{\partial_{\eta} \phi\left(., u_{n}\right)}\left(u_{n}\right)\right)\right\}\right\}\|+\|\left\{(1-\lambda) u_{n}+\lambda\left\{u_{n}-g\left(u_{n}\right)+\mathcal{R}_{I, \rho}^{\partial_{\eta} \phi\left(., u_{n}\right)}\left\{g\left(u_{n}\right)\right.\right.\right. \\
& \left.\left.-\rho\left(G\left(s_{n}, u_{n}\right)-C_{I, \rho}^{\partial_{\eta} \phi\left(., u_{n}\right)}\left(u_{n}\right)\right)\right\}\right\}-(1-\lambda) x^{*} \\
& +\lambda\left[x^{*}-g\left(x^{*}\right)+\mathcal{R}_{I, \rho}^{\partial_{\eta} \phi\left(., x^{*}\right)}\left\{x^{*}-g\left(x^{*}\right)-\rho\left(G\left(t^{*}, x^{*}\right)-C_{I, \rho}^{\partial_{\eta} \phi\left(., x^{*}\right)}\left(x^{*}\right)\right)\right\}\right] \| \\
\leq & \chi_{n}+(1-\lambda)\left\|u_{n}-x^{*}\right\|+\lambda \| u_{n}-x^{*}-\left(g\left(u_{n}\right)-g\left(x^{*}\right) \|\right. \\
& +\lambda \| \mathcal{R}_{I, \rho}^{\partial_{\eta} \phi\left(., u_{n}\right)}\left\{g\left(u_{n}\right)-\rho\left(G\left(s_{n}, u_{n}\right)-C_{I, \rho}^{\partial_{\eta} \phi\left(., u_{n}\right)}\left(u_{n}\right)\right)\right\} \\
& \quad-\mathcal{R}_{I, \rho}^{\partial_{\eta} \phi\left(., x^{*}\right)}\left\{x^{*}-g\left(x^{*}\right)-\rho\left(G\left(t^{*}, x^{*}\right)-C_{I, \rho}^{\partial_{\eta} \phi\left(., x^{*}\right)}\left(x^{*}\right)\right)\right\} \| .
\end{aligned}
$$

Using the same arguments as for (15), we have

$$
\left\|u_{n}-x^{*}-\left(g\left(u_{n}\right)-g\left(x^{*}\right)\right)\right\| \leq \sqrt{1-2 \delta_{g}+\lambda_{g}^{2}}\left\|u_{n}-x^{*}\right\| .
$$

where $\lambda_{g}^{2}>\left(2 \delta_{g}-1\right)$.

Using the same arguments as for (16), we get

$$
\begin{aligned}
\| \mathcal{R}_{I, \rho}^{\partial_{\eta} \phi\left(., u_{n}\right)}\left\{g\left(u_{n}\right)-\rho\left(G\left(s_{n}, u_{n}\right)-C_{I, \rho}^{\partial_{\eta} \phi\left(., u_{n}\right)}\left(u_{n}\right)\right)\right\}-\mathcal{R}_{I, \rho}^{\partial_{\eta} \phi\left(., x^{*}\right)}\left\{g\left(x^{*}\right)-\rho\left(G\left(t^{*}, x^{*}\right)\right.\right. \\
\left.\left.-C_{I, \rho}^{\partial_{\eta} \phi\left(., x^{*}\right)}\left(x^{*}\right)\right)\right\} \| \\
=\| \mathcal{R}_{I, \rho}^{\partial_{\eta} \phi\left(., u_{n}\right)}\left\{g\left(u_{n}\right)-\rho\left(G\left(s_{n}, u_{n}\right)-C_{I, \rho}^{\partial_{\eta} \phi\left(., u_{n}\right)}\left(u_{n}\right)\right)\right\}-\mathcal{R}_{I, \rho}^{\partial_{\eta} \phi\left(., u_{n}\right)}\left\{g\left(x^{*}\right)\right. \\
\left.\quad-\rho\left(G\left(t^{*}, x^{*}\right)-C_{I, \rho}^{\partial_{\eta} \phi\left(., x^{*}\right)}\left(x^{*}\right)\right)\right\}+\mathcal{R}_{I, \rho}^{\partial_{\eta} \phi\left(., u_{n}\right)}\left\{g\left(x^{*}\right)-\rho\left(G\left(t^{*}, x^{*}\right)-C_{I, \rho}^{\partial_{\eta} \phi\left(., x^{*}\right)}\left(x^{*}\right)\right)\right\} \\
\quad-\mathcal{R}_{I, \rho}^{\partial_{\eta} \phi\left(., x^{*}\right)}\left\{g\left(x^{*}\right)-\rho\left(G\left(t^{*}, x^{*}\right)-C_{I, \rho}^{\partial_{\eta} \phi\left(., x^{*}\right)}\left(x^{*}\right)\right)\right\} \| \\
\leq \quad \frac{\tau}{\delta}\left\|g\left(u_{n}\right)-g\left(x^{*}\right)-\rho\left(G\left(s_{n}, u_{n}\right)-C_{I, \rho}^{\partial_{\eta} \phi\left(., u_{n}\right)}\left(u_{n}\right)\right)-\left(G\left(t^{*}, x^{*}\right)-C_{I, \rho}^{\partial_{\eta} \phi\left(., x^{*}\right)}\left(x^{*}\right)\right)\right\| \\
\quad+\mu^{\prime}\left\|u_{n}-x^{*}\right\| .
\end{aligned}
$$

Using the same calculation as for (21), we have

$$
\begin{aligned}
\| g\left(u_{n}\right)-g\left(x^{*}\right)-\rho( & \left.G\left(s_{n}, u_{n}\right)-G\left(t^{*}, x^{*}\right)-\left(C_{I, \rho}^{\partial \phi\left(., u_{n}\right)}\left(u_{n}\right)-C_{I, \rho}^{\partial \phi\left(., x^{*}\right)}\left(x^{*}\right)\right)\right) \| \\
& \leq \lambda_{g}\left\|x_{n}-x^{*}\right\|+\rho \theta_{1}\left\|x_{n}-x^{*}\right\|+\rho \theta_{2}\left\|x_{n}-x^{*}\right\| \\
& =\left(\lambda_{g}+\rho \theta_{1}+\rho \theta_{2}\right)\left\|x_{n}-x^{*}\right\| .
\end{aligned}
$$

Combining (27)-(29) in (26), we have

$$
\left\|u_{n+1}-x^{*}\right\|
$$

$$
\begin{aligned}
& \leq \chi_{n}+\left[(1-\lambda)+\lambda \sqrt{1-2 \delta_{g}+\lambda_{g}^{2}}+\left(\frac{\tau}{\delta} \lambda\left(\lambda_{g}+\rho \theta_{1}+\rho \theta_{2}\right)+\mu^{\prime}\right)\right]\left\|u_{n}-x^{*}\right\| \\
& =\chi_{n}+\zeta(\theta)\left\|u_{n}-x^{*}\right\| .
\end{aligned}
$$


It follows from condition (13) that $0 \leq \zeta(\theta) \leq 1$ and applying Lemma 3 it follows that if $\lim _{n \rightarrow \infty} \chi_{n}=0$, thus $\lim _{n \rightarrow \infty} u_{n} \rightarrow x^{*}$. Therefore, the iterative sequences $\left\{x_{n}\right\}$ and $\left\{t_{n}\right\}$ generated by iterative Algorithm 1 are $\mathcal{R}_{I, \rho}^{\partial_{\eta} \phi(\cdot, x)}$-Stable.

\section{Numerical Example}

We provide a numerical example in support of most of the concepts used in Theorem 3.

Example 1. We take $\mathcal{H}=\mathbb{R}$ and let $G: \mathbb{R} \times \mathbb{R} \rightarrow \mathbb{R}, \eta: \mathbb{R} \times \mathbb{R} \rightarrow \mathbb{R}, g: \mathbb{R} \rightarrow \mathbb{R}$ be the single-valued mappings, $R_{I, \rho}^{\partial_{\eta} \phi(., x)}: \mathbb{R} \rightarrow \mathbb{R}$ be the resolvent operator, $C_{I, \rho}^{\partial_{\eta} \phi(., x)}: \mathbb{R} \rightarrow \mathbb{R}$ be the generalized Cayley operator, $\phi: \mathbb{R} \times \mathbb{R} \rightarrow \mathbb{R} \cup\{+\infty\}$ be a functional and $F: \mathbb{R} \rightarrow C B(\mathbb{R})$ be a multi-valued mapping such that

$$
\begin{aligned}
& \text { (i) } G(t, x)=\frac{t-x}{5}, \text { (ii) } g(x)=x, \text { (iii) } \eta(g(x), y)=x-y, \\
& \text { (iv) } \phi(g(x), x)=(g(x))^{2}+3 x^{2}, \quad(v) F(x)=\left\{\frac{x}{3}\right\}, \forall t \in F(x), x, y \in \mathbb{R} \text {. }
\end{aligned}
$$

First we calculate resolvent operator $R_{I, \rho}^{\partial_{\eta} \phi(\cdot, x)}$ and generalized Cayley operator $C_{I, \rho}^{\partial_{\eta} \phi(\cdot, x)}(x)$ as: For $\rho=1$, we have

$$
R_{I, 1}^{\partial_{\eta} \phi(\cdot, x)}(x)=\left[\begin{array}{c}
{[x+8 x]^{-1}=[9 x]^{-1}=\frac{x}{9}} \\
\text { Further }
\end{array} \text { and } C_{I, 1}^{\partial_{\eta} \phi(\cdot, x)}(x)=\left[2 R_{I, 1}^{\partial_{\eta} \phi(\cdot, x)}-1\right](x)=\left[2 \frac{x}{9}-x\right]=-\frac{7 x}{9}\right. \text {. }
$$

Further, we show that

(i) $G$ is Lipschitz continuous in both the arguments,

$$
\begin{aligned}
\left\|G\left(t_{1}, y\right)-G\left(t_{2}, y\right)\right\| & =\left\|\frac{t_{1}-y}{5}-\frac{t_{2}-y}{5}\right\| \\
& \leq \frac{2}{5}\left\|t_{1}-t_{2}\right\| .
\end{aligned}
$$

Thus, $G$ is Lipschitz continuous in the first argument with constant $\lambda_{G_{1}}=\frac{2}{5}$.

Similarly, we can show that $G$ is Lipschitz continuous in the second argument with constant $\lambda_{G_{2}}=\frac{2}{5}$.

(ii) $F$ is $\mathcal{D}$-Lipschitz continuous,

$$
\begin{aligned}
D(F(x), F(y)) & =\max \left\{\operatorname{Sup}_{x \in F(x)} d(x, F(y)), \operatorname{Sup}_{y \in F(y)} d(F(x), y)\right\} \\
& =\max \left\{\left|\frac{x}{3}-\frac{y}{3}\right|,\left|\frac{x}{3}-\frac{y}{3}\right|\right\} \\
& \leq \frac{1}{2}|x-y| .
\end{aligned}
$$

Thus, $F$ is $\mathcal{D}$-Lipschitz continuous with constant $\lambda_{D_{F}}=\frac{1}{2}$.

(iii) As $g(x)=x$, clearly $g$ is strongly monotone and Lipschitz continuous with constants $\lambda_{g}=1$ and $\delta_{g}=1$, respectively.

(iv) As $\phi(g(x), x)=(g(x))^{2}+3 x^{2}=x^{2}+3 x^{2}=4 x^{2}$.

Obviously, $\phi$ is lower semicontinuous and $\eta$-subdifferentiable, because $\partial_{\eta} \phi(g(x), x)=8 x$.

(v) clearly $\eta$ is strongly monotone and Lipschitz continuous with constants $\delta=1$ and $\tau=1$, respectively, and also $\eta(x, y)=-\eta(y, x)$.

(vi) As $h(y, u)=(x-u)(y-u)$, using the techniques of Ding [30] it is easy to show that $h$ is $0-D Q C V$ in $y$.

(vii) Under the above observations, we show the convergence of the sequences $\left\{x_{n}\right\}$ and $\left\{t_{n}\right\}$ generated by the Algorithm 1. 
For $\lambda=\frac{1}{2}$, the iterative sequence $\left\{x_{n}\right\}$ generated by Algorithm 1 becomes:

$$
x_{n+1}=\frac{x_{n}}{2}+\frac{1}{2}\left[\frac{x_{n}}{9}-\frac{87 x_{n}}{1215}\right]=0.519 x_{n}
$$

which clearly converges to 0 . By condition (10), it is obvious that the sequence $\left\{t_{n}\right\}$ also converge to 0 .

\section{Conclusions}

We have introduced and study a quite new problem called variational-like inequality problem involving Cayley operator in a real Hilbert space. Applying $\eta$-subdifferentiablity, we have shown that variational-like inequality problem involving Cayley operator is equivalent to a fixed point equation. This fixed point formulation is used to construct an iterative algorithm to obtain the solution of variational-like inequality problem involving a Cayley operator. Convergence and stability analysis are discussed, separately.

We remark that our results may be further extended in higher-order dimensional spaces.

Author Contributions: All the authors have contributed equally to this paper. All authors have read and agreed to the published version of the manuscript.

Funding: The research of C.-F.W. was funded by the Ministry of Science and Technology, Taiwan under Grant Number 109-2115-M-037-001.

Acknowledgments: The authors are thankful to the reviewers for their valuable suggestions which improve this article.

Conflicts of Interest: The authors declare no conflict of interest.

\section{References}

1. Stampacchia, G. Formes bilineaires coercivites sur les ensembles convexes. C. R. Acad. Paris 1964, 258, $4413-4416$.

2. Fichera, G. Problemi elastostatici con vincoli unilaterali: Il problema di Signorini con ambique condizione al contorno. Atti. Acad. Naz. Lincei. Mem. Cl. Sci. Nat. Sez. Ia 1963-1964, 7, 91-140.

3. Adly, S.; Oettli, W. Solvability of Generalized Nonlinear Symmetric Variational Inequalities; University of Mannheim: Mannheim, Germany, 1997.

4. Attouch, H.; Thera, M. A general duality principle for the sum of two operators. J. Convex Anal. 1996, 3, 1-24.

5. Baiocchi, C.; Capelo, A. Variational and Quasivariational Inequalities, Applications to Free Boundary Problems; Wiley: New York, NY, USA, 1984.

6. Brezis, H. Operateur Maximaux Monotone et Semigroupes de Contractions dans les Dspaces de Hilbert; North-Holland: Amsterdam, The Netherlands, 1973.

7. Cottle, R.W.; Giannessi, F.; Lions, J.L. Variational Inequalities: Theory and Applications; J. Wiley and Sons: New York, NY, USA, 1980.

8. Chang, S.S.; Wen, C.F.; Yao, J.C. Zero point problem of accretive operators in Banach spaces. Bull. Malays. Math. Sci. Soc. 2019, 42, 105-118. [CrossRef]

9. Fulga, C.; Preda, V. Nonlinear programming with $\psi$-preinvex and local $\psi$-preinvex functions. Eul. J. Oper. Res 2009, 192, 737-743. [CrossRef]

10. Fang, Y.P.; Huang, N.J.; Thompson, H.B. A new system of variational inclusions with $(H, \eta)$-monotone operators in Hilbert spaces. Comput. Math. Appl. 2005, 49, 365-374. [CrossRef]

11. Giannessi, F.; Maugeri, A. Variational Inequalities and Network Equilibrium Problems; Plenum Press: New York, NY, USA, 1995.

12. Glowinski, R.; Lions, J.; Tremolieres, R. Numerical Analysis of Variational Inequalities; North-Holland: Amsterdam, The Netherlands, 1981.

13. Nguyen, L.V.; Ansari, Q.H.; Qin, X. Weak sharpness and finite convergence for solutions of nonsmooth variational inequalities in Hilbert spaces. Appl. Math. Optim. 2020. [CrossRef]

14. Xu, Z.; Wang, Z. A generalized mixed variational inclusion involving $(H(.,),. \eta)$-monotone operators in Banach spaces. J. Math Res. 2010, 2, 47-56. [CrossRef]

15. Lions, J.L.; Stampacchia, G. Variational inequalities. Commun. Pure Appl. Math. 1967, 20, 493-512. [CrossRef]

16. Abbas, M.; Ibrahim, Y.; Khan, A.R.; Sen, M.D.L. Strong convergence of a system of generalized mixed equilibrium problem, splits variational inclusion problem and fixed point problem in Banach spaces. Symmetry 2019, 11, 722. [CrossRef]

17. Cho, S.Y. A monotone Bregman projection algorithm for fixed point and equilibrium problems in a reflexive Banach space. Filomat 2020, 34, 1487-1497. [CrossRef] 
18. Cho, S.Y. Implicit extragradient-like method for fixed point problems and variational inclusion problems in a Banach space. Symmetry 2020, 12, 998. [CrossRef]

19. Ceng, L.C. Hybrid viscosity extragradient method for systems of variational inequalities, fixed points of nonexpansive mappings, zero points of accretive operators in Banach spaces. Fixed Point Theory 2018, 19, 487-502. [CrossRef]

20. Guo, J.S.; Yao, J.C. Extension of strongly nonlinear quasivariational inequalities. Appl. Math. Lett. 1992, 5, 35-38. [CrossRef]

21. Harker, P.T.; Pang, J.S. Finite-dimensional variational inequality and nonlinear complementarity problems. Math. Program 1990, 48, 161-220. [CrossRef]

22. Hanson, M.A. On sufficiency of the Kuhn-Tucker conditions. J. Math. Anal. Appl. 1981, 80, 545-550. [CrossRef]

23. Hammad, H.A.; Rehman, H.; Sen, M.D.L. Advanced algorithms and common solutions to variational inequalities. Symmetry 2020, 12, 1198. [CrossRef]

24. Kinderlehrer, D.; Stampacchia, G. An Introduction to Variational Inequalities and their Applications; Academic Press: New York, NY, USA, 1980.

25. Kumam, W.; Muangchoo, K. Inertial iterative self-adaptive step size extragradient-like methods for solving equilibrium problems in real Hilbert space with applications. Axioms 2020, 9, 127. [CrossRef]

26. Moudafi, A.; Lehdili, N. From progressive decoupling of linkages in variational inequalities to fixed-point problems. Appl. Set Valued Anal. Optim. 2020, 2, 159-173.

27. Parida, J.; Sahoo, M.; Kumar, A. A Variational-like Inequality Problem. Bull. Austral. Math. Soc. 1989, 39, 225-231. [CrossRef]

28. Rathee, S.; Swami, M. Strong convergence of a hybrid method for infinite family of nonexpansive mapping and variational inequality. J. Indones. Math. Soc. 2021, 27, 90-102. [CrossRef]

29. Yao, J.C. Existence of generalized variational inequalities. Oper. Res. Lett. 1994, 15, 35-40. [CrossRef]

30. Ding, X.P. Generalized quasi-variational-like inclusions with nonconvex functionals. Appl. Math. Comput. 2001, 122, 267-282. [CrossRef]

31. Ding, X.P.; Tan, K.K. A minimax inequality with applications to existence of equilibrium point and fixed point theorem. Coll. Math. 1992, 63, 233-247. [CrossRef]

32. Zhou, X.J.; Chen, G. Diagonal convexity conditions for problems in convex analysis and quasivariational inequalities. J. Math. Anal. Appl. 1988, 132, 213-225. [CrossRef]

33. Noor, M.A. Nonconvex functions and variational inequalities. J. Optim. Theory Appl. 1995, 87, 615-630. [CrossRef]

34. Berinde, V. Iterative Approximation of Fixed Points: Lecture Notes in Mathematics 1912; Springer: Berlin/Heidelberg, Germany, 2007.

35. Ding, X.P. Perturbed proximal point algorithms for generalized quasi variational inclusions. J. Math. Anal. Appl. 1997, 210, 88-101. [CrossRef]

36. Ali, I.; Ahmad, R.; Wen, C,F. Cayley Inclusion Problem Involving XOR-operation. Mathematics 2019, 7, 302. [CrossRef]

37. Ding, X.P.; Luo, C.L. Perturbed proximal point algorithms for general quasi-variational-like inclusions. J. Comput. Appl. Math. 2000, 113, 153-165. [CrossRef]

38. Hassouni, A.; Moudafi, A. A perturbed algorithm for variational inclusions. J. Math. Anal. Appl. 1994, 185, 706-712. [CrossRef]

39. Huang, N.J. Generalized nonlinear variational inclusions with noncompact valued mappings. Appl. Math. Lett. 1996, 9, 25-29. [CrossRef]

40. Siddiqi, A.H.; Ansari, Q.H. Strongly nonlinear quasivariational inequalities. J. Math. Anal. Appl. 1990, 149, 444-450. [CrossRef]

41. Siddiqi, A.H.; Ansari, Q.H. General strongly nonlinear variational inequalities. J. Math. Anal. Appl. 1992, 166, 386-392. [CrossRef]

42. Nadler, S.B. Multivalued contraction mapping. Pac. J. Math. 1969, 30, 475-488. [CrossRef] 